\title{
DETERMINAÇÃO DO COMPRIMENTO E GRAU DE CURVATURA EM CANAIS RADICULARES CURVOS SIMULADOS CONFECCIONADOS EM BLOCOS DE RESINA FENÓLICA
}

\section{DETERMINING THE LENGTH AND DEGREE OF CURVATURE IN SIMULATED CURVED ROOT CANALS IN A PHENOLIC RESIN BLOCS}

\author{
João Marcelo Ferreira de Medeiros * \\ Gabriela de Almeida Rodrigues * \\ Alexandre Cursino de Moura Santos \\ Luiz Carlos Laureano da Rosa \\ Pedro Luiz de Carvalho "mon \\ Evandro Luís Nohara
}

\begin{abstract}
RESUMO
Introdução:É difícil encontrar canais radiculares curvos de dentes humanos com mesmo grau de curvatura. O propósito desta pesquisa é determinar, valendo-se de um cilindro construído a partir de cálculo matemático trigonométrico o ângulo de fios metálicos que foram moldados neste cilindro e posterior determinação de 75 graus de curvatura e comprimento de 21 milímetros desses fios metálicos e dos canais radiculares simulados depois da confecção dos blocos de resina fenólica, após avaliação radiográfica. Métodos: Foi construído um dispositivo com formato de cilindro para que o fio fosse envolvido no sulco do cilindro, obtendo-se arranjo do arco com o ângulo pretendido. Foi realizada uma tomada radiográfica do arco metálico e a determinação do grau de curvatura conforme método de Schneider (1971), obtendo-se os ângulos. Após inclusão dos fios metálicos nos blocos em uma unidade de embutimento, nova tomada radiográfica e novo traçado para obtenção do ângulo dos canais simulados. Os dados foram tabelados e feita análise estatística com média aritmética, desvio-padrão e teste " $\mathrm{t}$ " Student. Resulados e Conclusóes: O teste "t" Student para amostras relacionadas aponta Pvalor $<0,05$, indicando que ocorreu diferença estatisticamente significante entre os ângulos (graus de curvatura) antes e depois da inclusão ao nível de $95 \%$ de confiança. A obtenção do arco metálico no cilindro nas duas condiçóes bem como o traçado para determinação do ângulo na radiografia representam uma ferramenta reproduzível assim como um método correto e válido na determinação do grau de curvatura.
\end{abstract}

DESCRITORES: Tratamento do canal radicular $\bullet$ Endodontia $\bullet$ Fenóis $\bullet$ Teste de materiais $\bullet$ Radiografia.

\section{ABSTRACT}

Introduction: It is hard to find curved root canals of human teeth with the same degree of curvature. The purpose of this study is to determine worth is a cylinder constructed from mathematical calculation of the angle Trigonometric wires that were molded in cylinder and subsequent determination of 75 degrees of curvature of $21 \mathrm{~mm}$ and length of wires and channels simulated root after making the blocks of phenolic resin, after radiographic evaluation. Method: A device was built with cylinder format to be involved in the wire groove of the cylinder resulting in arrangement of the arc with the desired angle. Taking radiographic metal arc and determining the degree of curvature as method of Schneider (1971) yields the desired angles. After that, an inclusion of wires was made in blocks in an inlay radiographic and making new route for obtaining new angle of the simulated canals. Data were scheduled and performed statistical analysis as arithmetic mean, standard deviation and test " $\mathrm{t}$ " Student. Results and conclusions: The test "t" Student points related to samples Pvalor $<0.05$ indicating that there was a statistically significant difference between the angles (degrees of curvature) before after inclusion at $95 \%$. The acquisition of the arc in the metal cylinder in the two conditions and the route to determine the angle in radiography is a reproducible tool as well as correct and valid method for determining the degree of curvature.

DESCRIPTORS: Root canal therapy $\bullet$ Endodontics $\bullet$ Phenols $\bullet$ Materials testing $\bullet$ Radiography.

* Professor Assistente Doutor da Disciplina de Endodontia do Departamento de Odontologia da Universidade de Taubaté (UNITAU), Taubaté/SP. Professor do Programa de Pós-Graduação da Universidade de Taubaté (UNITAU), Taubaté/SP jmedeiros@unitau.br

** Acadêmica do Departamento de Odontologia da Universidade de Taubaté (UNITAU), Taubaté/SP. rodrigues.gabizinha@gmail.com

*** Professor Assistente da Disciplina de Clínica Integrada do Departamento de Odontologia da Universidade de Taubaté (UNITAU), Taubaté/SP. assantos@ bighost.com.br

**** Professor Assistente Doutor da Disciplina de Bioestatística do Departamento de Matemática e Física da Universidade de Taubaté (UNITAU), Taubaté/SP. laureanodarosa@gmail.com

***** Professor Assistente Doutor da Disciplina de Imaginologia Dentomaxilofacial do Departamento de Odontologia da Universidade de Taubaté (UNITAU), Taubaté/SP. pedrolc@unitau.br

******* Professor Assistente Doutor da Disciplina de Tecnologia dos Materiais do Departamento de Engenharia Mecânica da Universidade de Taubaté. Coordenador do Laboratório de Materiais e Ensaios e Vice-Coordenador do Programa de Mestrado Profissional em Engenharia Mecânica da Universidade de Taubaté (UNITAU), Taubaté/SP. evandro@unitau.br 
Medeiros JMF, Rodrigues GA, Santos ACM, Rosa LCL, Carvalho PL, Nohara EL. Determinação do comprimento e grau de curvatura em canais radiculares curvos simulados confeccionados em blocos de resina fenólica. Revista de Odontologia da Universidade Cidade de São Paulo 2009 set-dez; 21(3): 202-11

\section{INTRODUÇÃO E REVISTA DA LITERATURA}

Existem diferentes substratos utilizados para avaliar a capacidade e eficiência de corte de instrumentos endodônticos, notadamente o dente, o osso bovino, a resina acrílica e a fenólica.

Recomenda-se muito a utilização de dentes naturais humanos extraídos (Medeiros et al. ${ }^{23}$ 2000, Medeiros et al. ${ }^{21}$ 2002, Medeiros et al. ${ }^{20}$ 2006, Medeiros et al. ${ }^{22}$ 2006) e embora pesquisadores realizem suas pesquisas com osso bovino (Souza et al. ${ }^{34}$ 1998, Silva ${ }^{32}$ 2001, Claro ${ }^{8} 2004$ ), e há aqueles que utilizaram materiais sintéticos resinosos (Borges $^{6}$ 2005, Sakane ${ }^{28}$ 2007, Gonçalves ${ }^{11}$ 2007, AlvesClaro et al. ${ }^{24}$ 2008, Medeiros et al. ${ }^{3}$ 2008).

A vantagem da utilizaçáo da resina, por representar amostras com geometria e dureza semelhantes entre si, é permitir a confecção de canais com graus de curvatura semelhantes propiciando padronização melhor da pesquisa. Contrariamente seria difícil colher dentes com graus de dureza semelhantes, para um modelo de investigação (Schäfer e Tepel $\left.{ }^{29} 1996\right)$.

Schneider ${ }^{31}$ (1971) realizou experimento objetivando a determinação da frequência de preparos de forma circular que poderiam ser produzidos no terço apical de canais radiculares retos e curvos através de instrumentação manual. Para tanto, o pesquisador constituiu uma amostra de 27 caninos inferiores e superiores, 1 incisivo lateral superior e 1 pre-molar inferior - dentes extraídos, permanentes, unirradiculares e com rizogênese completa. Após a secção das coroas, os comprimentos radiculares foram padronizados em 15 milímetros a partir do ápice. Em seguida todos os espécimes foram radiografados e amensuração do grau de curvatura de cada uma das raízes foi realizada segundo metodologia desenvolvida pelo próprio pesquisador. Então, as raízes foram classificadas da seguinte forma: (I) retas: 5 graus ou menos; (II) moderada: de 10 a 20 graus e (III) severa: de 25 a 70 graus. Os canais foram preparados exclusivamente com limas em movimentos de alargamento. Obturadas as raízes, foram seccionadas transversalmente, avaliadas sob microscopia de dissecção (x25), e algumas amostras representativas foram fotografadas. Os resultados demonstraram que os canais retos são muito mais prontamente preparados em forma circular que os canais curvos. Os preparos circulares foram obtidos em $51 \%$ das vezes ao nível de 1 milímetro do ápice comparados com 17\% a 5 milímetros.

Glosson et al. ${ }^{10}$ (1995), Schäfer ${ }^{30}(1996)$, Szep et al..$^{35}$
(2001), Fariniuk et al. ${ }^{9}$ (2001), Hulsmann et al. ${ }^{14}$ (2003), Hulsmann et al. ${ }^{15}$ (2003), Ankrum et al. ${ }^{4}$ (2004), Song et $a l^{33}$ (2004), Veltri et al. ${ }^{37}$ (2004), Calberson et al. ${ }^{7}$ (2004), Veltri et al. ${ }^{35}$ (2005), Guelzow et al. ${ }^{13}$ (2005), Yoshimine et al. ${ }^{39}$ (2005), Plotino et al. ${ }^{26}$ (2006), Grande et al. ${ }^{12}$ (2006), Al-Sudani e Al-Shahrani ${ }^{2}$ (2006), Plotino et al. ${ }^{27}$ (2007), Javaheri e Javaheri ${ }^{16}$ (2007), Yang et al. ${ }^{38}$ (2007), Mahran e AboEl-Fotouh ${ }^{18}$ (2008), Johnson et al. ${ }^{17}$ (2008) são unânimes em comparar diferentes instrumentos de níquel-titânio e aço inoxidável em substratos de canais radiculares curvos, simulados ou em dentes humanos, cuja variação do ângulo de curvatura está situada entre 150 a 750.

Ademais, os referidos autores analisam em suas pesquisas o transporte do canal e sua centralização, remoção de quantidade de dentina e produçáo de formas mais circulares do canal, sem degraus e transporte, técnicas de preparo de canais, fratura do instrumento, manutenção do comprimento de trabalho, média de tempo de trabalho, limpeza das paredes do canal radicular, motor de alto torque, incidência de ruptura e distorçóes de limas, distorçóes na lâmina de corte dos instrumentos como trincas de limas, formação de zip ou desvio no final da preparação, menor flexibilidade da porção terminal do instrumento, fadiga cíclica de instrumentos, influência do raio de curvatura, ação de raspagem do instrumento, desvio apical, segurança de trabalho (falha do instrumento, bloqueio apical e perda de tempo de trabalho), capacidade de modelar (retificação, área da seção transversal, transporte e centralidade do preparo), pequena conicidade e torção.

A resina acrílica é o material de escolha nestas investigaçóes, ao contrário do uso de substratos à base de resina fenólica (Baquelite ${ }^{\circledast}$ ) que a propósito trata-se de modelo experimental de eficácia, apesar de o primeiro estudo com instrumentos endodônticos ter sido realizado no final da década de 80 e somente a partir do século XXI é que surgiu literatura com algumas investigaçóes, usando-se esse material em substratos à base de resina fenólica.

Aliás, Morrinson et al. ${ }^{25}$ (1989) analisando esterilização e eficiência de corte de instrumentos, utilizando como substratos dentes humanos e placas sulcadas de resina fenólica e realizando neste último grupo o procedimento de uso linear contínuo mecanizado. Observaram os autores que, a resina fenólica usada nesta pesquisa constitui um excelente substrato para o teste de corte. 
Medeiros JMF, Rodrigues GA, Santos ACM, Rosa LCL, Carvalho PL, Nohara EL. Determinação do comprimento e grau de curvatura em canais radiculares curvos simulados confeccionados em blocos de resina fenólica. Revista de Odontologia da Universidade Cidade de São Paulo 2009 set-dez; 21(3): 202-11

Borges6 (2005) analisou comparativamente a capa cidade de corte, por meio de análises em um projetor de perfil, de duas limas, sendo uma de secção quadrangular e a outra, de secção triangular em liga de aço inoxidável, ambas adaptadas a movimento linear com atuação padronizada sobre placas de resina fenólica. Comparando as marcas comerciais Maillefer (Ballaigues, Swiss) e FKG (FKG, Dentaire, Swiss), esta última apresentou maior capacidade de corte.

Sakane ${ }^{28}$ (2007) objetivou comparar a capacidade de corte e o desgaste, de limas endodônticas manuais de níquel-titânio. Para tanto, utilizou dez instrumentos de níquel-titânio das marcas Maileffer-Dentsply, FKG e Densell, todas de número 35, acopladas a um dispositivo de ensaio de desgaste que realizava movimentos contínuos e lineares em placas sulcadas à base de resina fenólica. Concluiu-se que os instrumentos apresentaram capacidades de cortes semelhantes sem diferenças estatísticas significativas. Quanto ao desgaste, verificou-se que as lâminas de corte da marca Densell deformaramse menos, comparativamente aos instrumentos da marca Dentsply e FKG; porém, entre essas duas últimas, não houve diferença estatística significante.

Gonçalves ${ }^{11}$ (2007) analisou a capacidade de corte e deformação das limas K3 e RT Densell, utilizando 50 blocos com canais radiculares simulados confeccionados em resina fenólica. As limas de diâmetro da ponta número 20 e conicidade 0.02 usadas foram avaliadas por meio de microscópio com iluminação episcópica, em campo escuro Nikon Epiphot 200. Os resultados revelaram que as limas de níquel-titânio do sistema rotatório da marca RT Densell ${ }^{\oplus}$ possuem maior capacidade de corte do que as limas do sistema rotatório da marca $\mathrm{K} 3$, com significado estatístico. Considerando-se a superfície das lâminas de corte observou-se deformação sem diferença estatística entre as marcas.

Alkmin et al. ${ }^{1}$ (2007) detalharam o mesmo por meio de fotografia de bases construídas com placas e blocos com canais simulados à base de resina fenólica. A construção dos blocos foi feita tomando-se uma parte do pó da resina granulada pesada e depositada no fundo da matriz do aparelho termo-plastificador. Um fio ortodôntico de número 0,6 foi colocado sobre esta porção de pó e sobre este uma nova porção do pó de resina, acionandose o termo-plastificador e com uma carga de $20-25 \mathrm{kN}$, tempo de 10-12 minutos e temperatura situada entre 1900C a 2100C obtevendo-se cilindros de 8 milímetros de altura e 30 milímetros de diâmetro, os quais foram lixados realizando-se os acabamentos.

Medeiros et al. ${ }^{24}$ (2008) cotejaram a capacidade de corte de limas Flexofile em 20 canais simulados retos confeccionados em blocos de resina fenólica. Em um grupo usou limas Flexofile manualmente de número 15 ao 40 e, no outro, preparo do canal com limas Flexofile (15-40) acopladas ao contra-ângulo no aparelho Endo-Griper. Não houve diferença quanto à capacidade de corte, comparadas duas técnicas de instrumentaçáo, sendo que em ambas as técnicas as limas perderam a capacidade de corte segundo o número de uso.

Alves-Claro et al. ${ }^{3}$ (2008) investigaram a resistência ao desgaste de instrumentos segundo movimentos de vai-vem de duas limas: a Flexofile (\#35) e a Nitiflex (\#35), permitindo ciclo de desgaste sobre placas sulcadas de Baquelite ${ }^{\ominus}$. Determinaram a flexibilidade das limas submetendo-as à implantaçáo iônica por imersão em plasma, avaliando os efeitos deste tratamento. A microdureza no núcleo das limas e das placas de desgaste foi ensaiada pelo método de Vickers. As placas de Baquelite ${ }^{\bullet}$ são, sem sombra de dúvida, um possível substituto do osso bovino úmido sem apresentar inconvenientes.

A resistência ao desgaste das limas de níquel-titânio foi significativamente maior que das limas de aço inoxidável e de níquel-titânio não tratadas.

Dentre as qualidades e propriedades desse substrato, destacam-se dureza homogênea em todas as amostras semelhante ao osso bovino úmido, excelente substrato para uso em teste de penetração, baixa higroscopia, boa estabilidade dimensional, baixo custo de preparação, reprodutibilidade controlável na preparação das amostras e perda de peso em resposta ao ciclo de limagem (AlvesClaro et al. ${ }^{3}$ 2008; Medeiros et al..$^{24}$ 2008).

Claro $^{8}$ (2004) demonstra os valores calculados de dureza Vickers sendo para as placas de osso de fêmur bovino úmido valor de 39,72, para a baquelita, índice de 39,92, resina acrílica (polimetilmetacrilato) cerca de 21,10 e placas de osso bovino desidratado, da ordem de 64,85 . Mais um motivo para escolha da baquelita, visto que apresenta dureza Vickers próxima à dentina humana $\left(57-60 \mathrm{Kg} / \mathrm{mm} 2\right.$ ) (Anusavice e Antonson $\left.{ }^{38} 2005\right)$ quando comparada à resina acrílica.

O propósito desta pesquisa é determinar, valendo-se de um cilindro construído a partir de cálculo matemático trigonométrico, o ângulo de fios metálicos que foram moldados nesse cilindro e posterior determinação de 
Medeiros JMF, Rodrigues GA, Santos ACM, Rosa LCL, Carvalho PL, Nohara EL. Determinação do comprimento e grau de curvatura em canais radiculares curvos simulados confeccionados em blocos de resina fenólica. Revista de Odontologia da Universidade Cidade de São Paulo 2009 set-dez; 21(3): 202-11

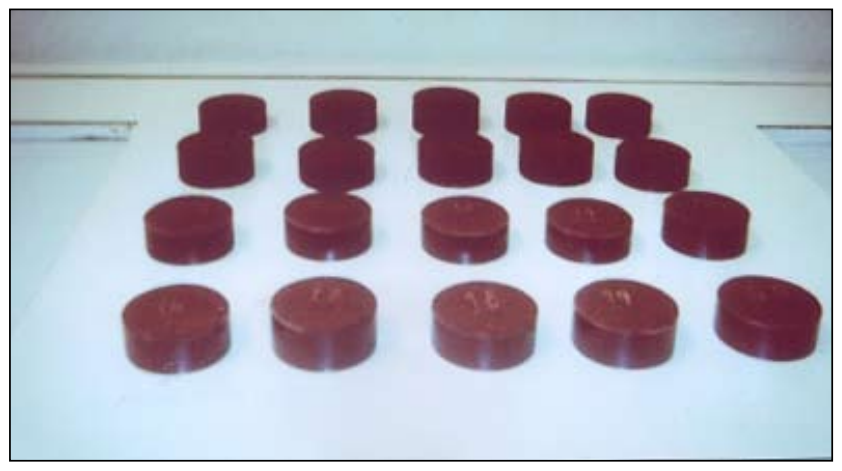

Figura 1: Blocos de baquelita

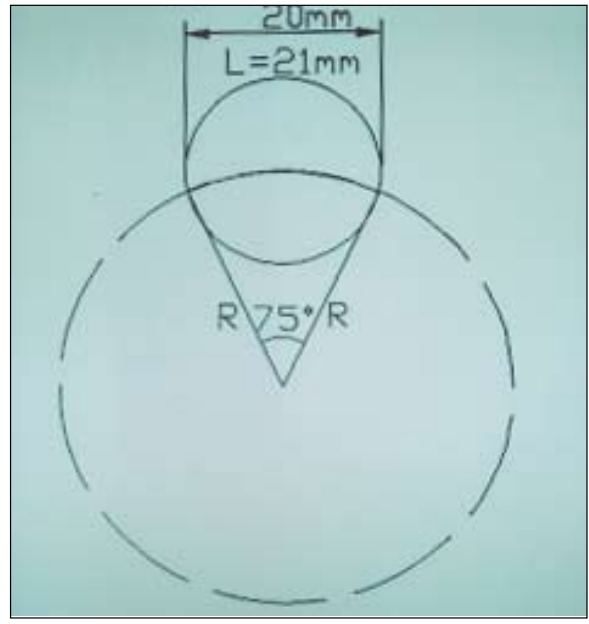

Figura 2: Determinaçâo do arco

75 graus de curvatura e comprimento de 21 milímetros desses fios metálicos e dos canais radiculares simulados depois da confecção dos blocos de resina fenólica, após avaliação radiográfica.

\section{MATERIAIS E MÉTODOS}

Utilizaram-se 20 blocos de baquelita (Figura 1) com canais curvos simulados em resina fenólica "Multfast Brown" (marca Struers/EUA) fabricados pelo Laboratório de Materiais, Tratamentos de Superfície e Nanotecnologia do Departamento de Engenharia Mecânica da Universidade de Taubaté.

A fabricação dos blocos inclui procedimentos de embutimento (Manual de operação e manutenção. Pantec ${ }^{19}$ 1992), lixamento e acabamento final os quais foram os mesmos observados para procedimentos e manufatura dos blocos e placas de resina fenólica de canais simulados (Borges ${ }^{6}$ 2005; Sakane ${ }^{28}$ 2007; Gonçalves ${ }^{11} 2007$; Alkmin et al. ${ }^{1}$ 2007; Alves-Claro et al. ${ }^{3}$ 2008; Medeiros et al. ${ }^{24}$ 2008).

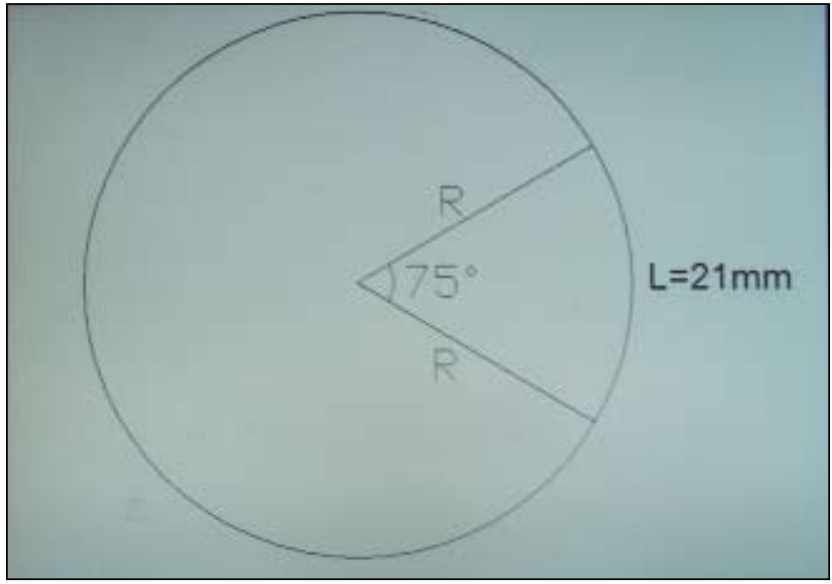

Figura 3 - Determinação da equivalência de graus em radianos

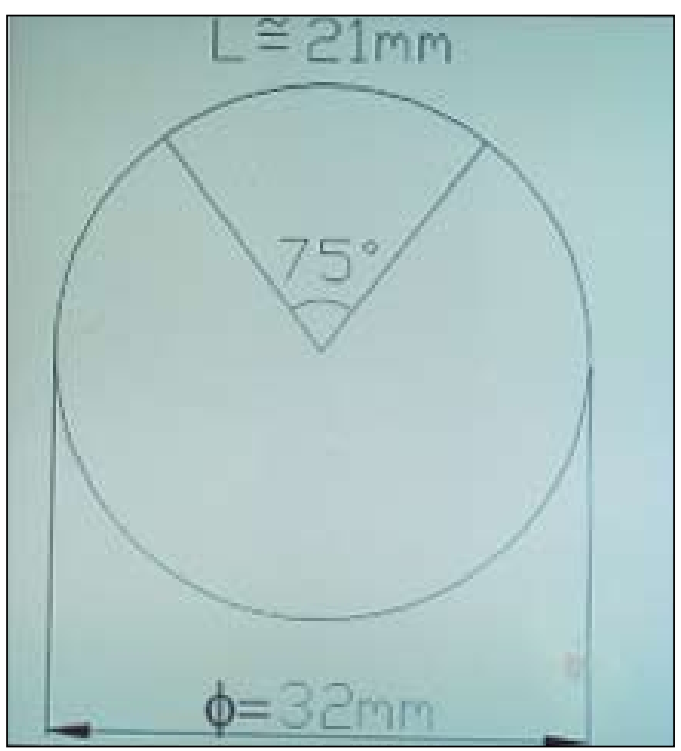

Figura 4: Método para construção do gabarito

Para obtençáo da curvatura do fio ortodôntico realizou-se sequência de procedimento a partir de cálculo trigonométrico, a saber: determinou-se como obter um arco de comprimento igual a 21 milímetros, com ângulo de 750 de curvatura, numa circunferência de 20 milímetros de diâmetro.

Para tanto, foi feita outra circunferência para obtenção de tal curvatura e do raio $\mathrm{R}$ conforme mostra a Figura 2.

Para se definir o raio $\mathrm{R}$, realizou-se como primeiro passo a determinação da equivalência de graus em radia$\operatorname{nos}(\theta)$ (Figura 3).

$$
\begin{aligned}
& 2 \tilde{I I ~ r a d}=3600 \\
& \theta=750
\end{aligned}
$$


Medeiros JMF, Rodrigues GA, Santos ACM, Rosa LCL, Carvalho PL, Nohara EL. Determinação do comprimento e grau de curvatura em canais radiculares curvos simulados confeccionados em blocos de resina fenólica. Revista de Odontologia da Universidade Cidade de São Paulo 2009 set-dez; 21(3): 202-11

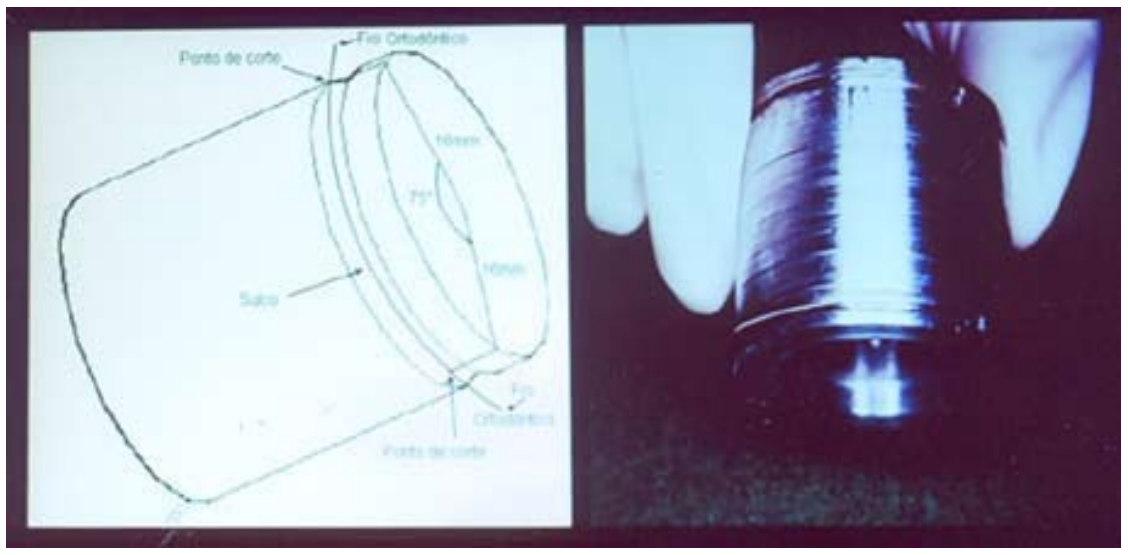

Figura 5: Perspectiva do cilindro
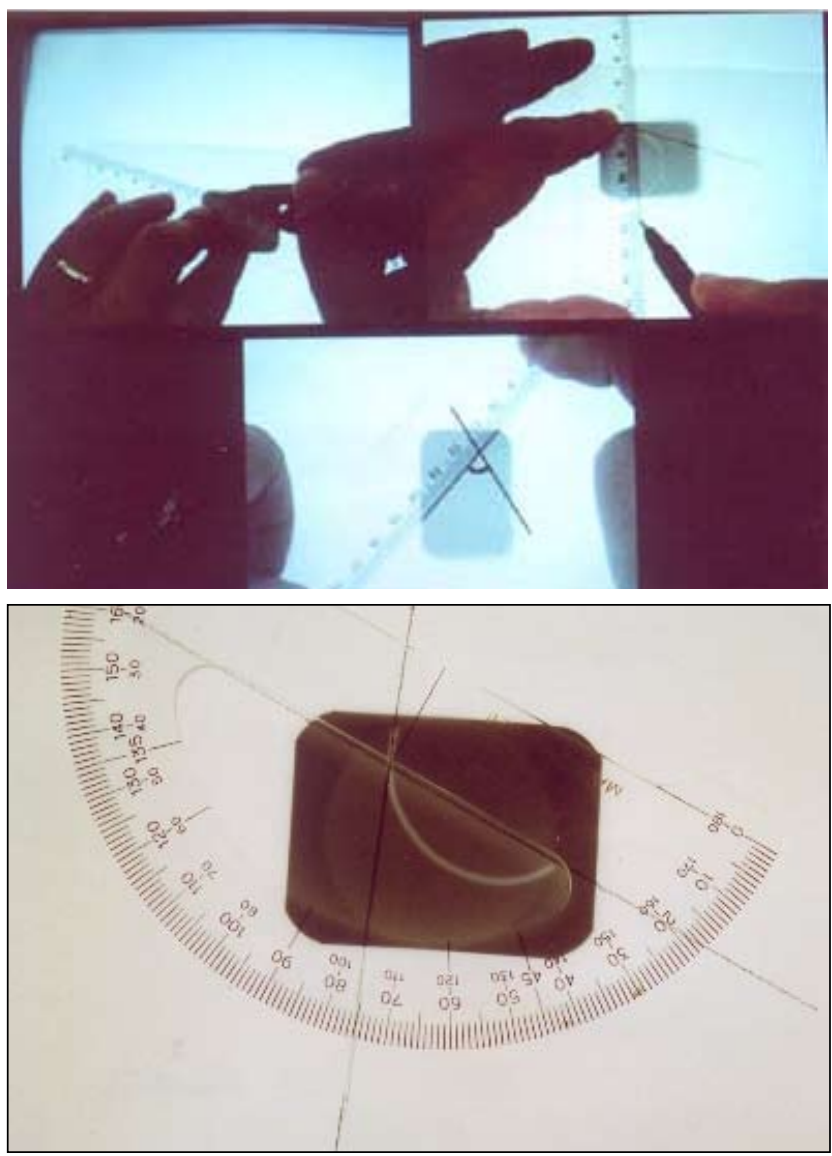

Figura 7: Traçado do grau de curvatura na radiografia

$360 . \theta=750.2 \mathrm{II}$

$\theta=1500 . \tilde{I I} / 3600$

$\theta=1,309 \mathrm{rad}$

A medida L da corda (fio) é função do raio e do arco, como segue:

$\mathrm{L}=\theta \cdot \mathrm{R}$ como $\mathrm{L}=21 \mathrm{~mm} \mathrm{e} \theta=1,309 \mathrm{rad}$ donde $\mathrm{R}=$

$\mathrm{L} / \theta$ ou seja $21 / 1,309$

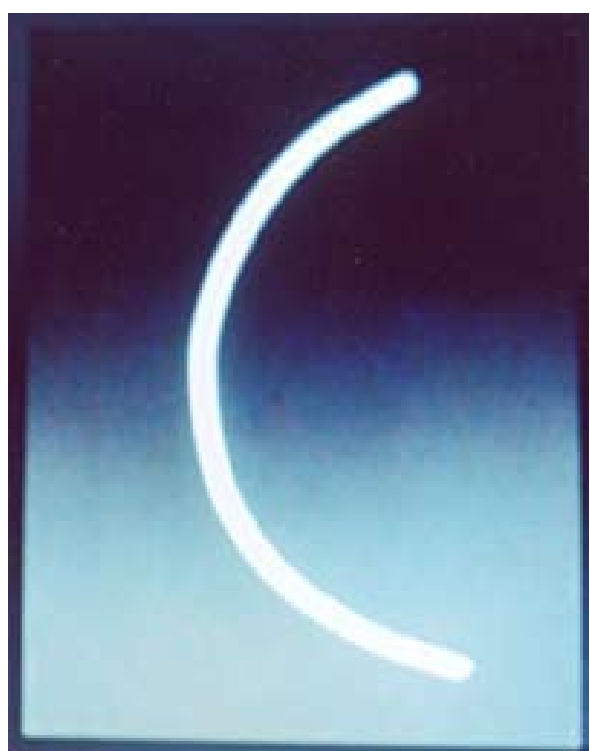

Figura 6: Radiografia do fio ortodôntico previamente encurvado

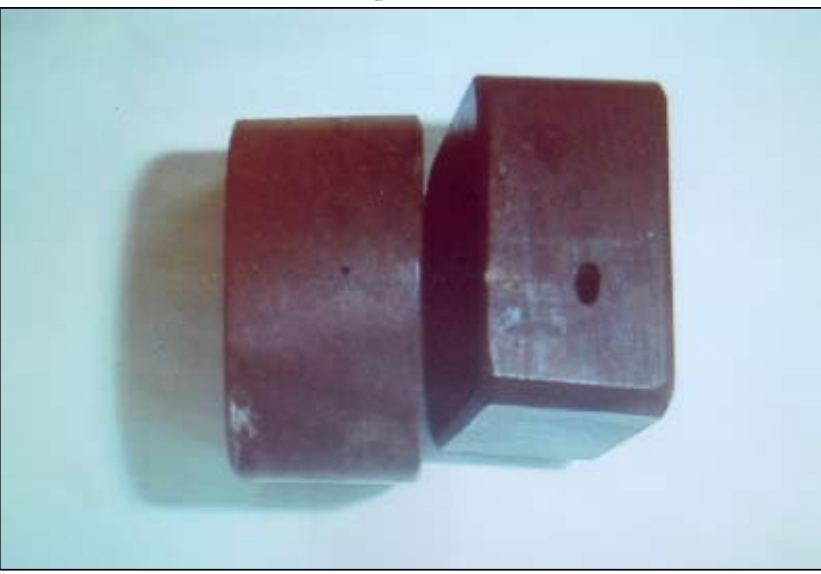

Figura 8: Bloco com canal simulado

Para construção do gabarito, construiu-se um disco ou cilindro cujo raio media 16 milímetros, ou seja, 32 milímetros de diâmetro (Figura 4)

A partir dos dados da Figura 4, construiu-se, de forma artesanal, um cilindro com curvatura e comprimento desejados (Figura 5) para que se pudessem obter grau de curvatura do fio ortodôntico e comprimento em milímetros do fio ortodôntico.

Uma vez obtido o cilindro, o fio ortodôntico será envolvido no sulco do cilindro, procedendo-se corte nas marcas indicadas "ponto de corte", obtendo-se dessa maneira, um fio ortodôntico com um comprimento (L) aproximado de 21 milímetros e curvatura de 750 .

$\mathrm{Na}$ sequência, foi realizada uma tomada radiográfica do fio ortodôntico antes de sua inclusão (Figura 6) com 
Medeiros JMF, Rodrigues GA, Santos ACM, Rosa LCL, Carvalho PL, Nohara EL. Determinação do comprimento e grau de curvatura em canais radiculares curvos simulados confeccionados em blocos de resina fenólica. Revista de Odontologia da Universidade Cidade de São Paulo 2009 set-dez; 21(3): 202-11

distância focal de 20 centímetros, tempo de exposição 0,16 segundos e processamento automático 5,5 minutos de seco a seco.

Processada a radiografia, realizou-se a determinação de grau de curvatura de acordo com o método de Schneider $^{31}$ (1971) et al. valeu-se de um lápis de ponta fina, papel vegetal, régua e transferidor. $\mathrm{O}$ papel vegetal foi colocado sobre a radiografia sendo, a seguir, traçada uma linha com auxílio de uma régua, calibrada em milímetros e lápis de ponta fina, paralela ao longo eixo do canal, isto é, traçado realizado em cima da parte reta do canal curvo.

Valendo-se novamente de uma régua foi feita uma segunda linha que se inicia num ponto situado a partir do término do canal do lado oposto até a intersecção com a primeira linha quando esta termina no canal curvo. Em continuidade, foram medidos os ângulos agudos formados pela intersecção dessas duas linhas, utilizando-se um transferidor (Figura 7).

Uma vez determinado o grau de curvatura em cada arco, foi anotado na Tabela 1 , considerando-se cada valor encontrado pelo traçado realizado.

Seguiram-se a colocação e prensagem na unidade de embutimento do arco de fio metálico e lixamento dos blocos (Manual de operação e manutenção. Pantec19 1992).

O bloco no final do acabamento tinha as seguintes dimensôes: 15 milímetros de altura, arco de comprimento igual a 21 milímetros de diâmetro, ângulo de 750 de curvatura numa circunferência de 20 milímetros de diâmetro (Figura 8).

Seguiu-se nova tomada radiográfica dos blocos com canais simulados curvos. Após isso, realizou-se novo traçado dos ângulos de curvatura de acordo com método de Schneider ${ }^{31}$ (1971), com vistas a determinar o seu grau de curvatura e anotados na Tabela 1 , considerando cada novo valor encontrado.

Foi executada análise estatística dos resultados obtidos, valendo-se de estatística descritiva, mostrando-se o comportamento dos dados por meio de média aritmética e desvio-padrão. Para a inferência estatística foi utilizado teste paramétrico " $\mathrm{t}$ " Student para dados relacionados, com nível de significância de 5\% na comparação de duas amostras.

\section{RESULTADOS}

Os resultados da pesquisa estão expressos na Tabela 1.
Nessa Tabela, os valores relativos às médias, desviopadrão e coeficiente de variaçấo obtidos antes da inclusão dos arcos bem como após sua inclusão estão expressos em graus, não apresentando significado estatístico entre as duas tomadas.

Tabela 1 - Média (), Desvio-padrão ( $\sigma$ ) e Coeficiente de Variação $(\mathrm{CV})$ dos grupos em relação à grandeza grau de curvatura

\begin{tabular}{cccc}
\hline \hline \multicolumn{4}{c}{ GRAU DE CURVATURA } \\
\hline GRUPO & $\mathrm{x}$ & $\sigma(\mathrm{grau})$ & $\mathrm{CV}(\%)$ \\
ANTES & 74,80 & $\pm 1,74$ & 2,32 \\
DEPOIS & 74,20 & $\pm 2,33$ & 3,14 \\
& \multicolumn{2}{c}{ Pvalor $=0,0485$} \\
\hline \hline
\end{tabular}

(significante, amostras diferentes, ao nível de 0,05 )

De acordo com a Tabela, antes da inclusão dos arcos o valor encontrado da média do ângulo de cada bloco foi de 74,80 e desvio-padráo de 1,74. Da mesma forma, após a inclusão dos arcos verificou-se ângulo médio de 74,20 e desvio-padrão de 2,33.

O teste " $\mathrm{t}$ " Student para amostras relacionadas aponta Pvalor $<0,05$ indicando que ocorreu diferença estatisticamente significante entre os ângulos (graus de curvatura) antes e depois da inclusão ao nível de $95 \%$ de confiança.

\section{DISCUSSÃO}

O preparo do canal radicular curvo representa etapa das mais importantes no tratamento endodôntico. É relevante analisar o tipo do instrumento utilizado, técnica de preparo e, indiscutivelmente, a destreza do operador.

A utilização de substratos como plásticos se torna cada vez mais evidente em pesquisas na endodontia, sobretudo, na instrumentaçáo de canais, de modo a valorizar o ensino e aprendizagem de forma adequada. Houve um avanço nos últimos 10 anos nos instrumentos à base de níqueltitânio dos mais variados tipos, marcas, empregando-se as mais diferentes técnicas de instrumentação, adequando-os aos sistemas rotatórios e também em bases para ensaios.

Substrato à base de resina fenólica e limas endodônticas têm sido utilizados, sobretudo em ensaios com blocos ou placas cujo objetivo é avaliar a capacidade de corte, eficiência de corte e deformação de instrumentos de aço inoxidável e níquel-titânio (Morrinson et al..$^{25}$;

Borges $^{6}$ 2005; Sakane ${ }^{28}$ 2007; Gonçalves ${ }^{11}$ 2007; Al- 
Medeiros JMF, Rodrigues GA, Santos ACM, Rosa LCL, Carvalho PL, Nohara EL. Determinação do comprimento e grau de curvatura em canais radiculares curvos simulados confeccionados em blocos de resina fenólica. Revista de Odontologia da Universidade Cidade de São Paulo 2009 set-dez; 21(3): 202-11

ves-Claro et al. ${ }^{3}$ 2008; Medeiros et al. ${ }^{24}$ 2008).

Aliás, a confecção de bloco à base de osso bovino é difícil, já que a feitura de canal no interior desse substrato é dispendioso em termos de tempo.

Entende-se que, embora a maioria dos substratos utilizados em laboratório sejam incapazes de reproduzir o dente natural humano, a resina fenólica possui propriedades físicas semelhantes aos dentes naturais humanos.

Sem contar a facilidade na confecção de blocos com canais simulados, ao contrário do osso bovino poderâo ser construídos de forma curva, inclusive em série, com mesmo grau de curvatura.

Escolher dentes humanos às vezes é fácil, embora a ocorrência de diferenças nesses dentes escolhidos em relação a sua natureza geométrica e dureza constitua um achado difícil.

Neste trabalho houve a intenção de criar condiçóes que permitam desenvolver pesquisa com a substância chamada resina fenólica.

E para tal valeu-se de cilindro construído a partir de cálculo matemático trigonométrico. Uma vez obtido o cilindro, foram moldados e radiografados fios metálicos com nova avaliação radiográfica depois da confecção dos blocos de resina fenólica. Diga-se a propósito que os dois casos deveriam apresentar 75 graus de curvatura e comprimento de 21 milímetros.

$\mathrm{Na}$ verdade, tal cálculo permitiu a confecção de um cilindro que serviu de gabarito para que fosse possível obter um arco metálico com dimensóes que serviram de suporte na determinação do ângulo pretendido (Figuras 2, 3, 4 e 5).

Assim é que, uma vez moldado o arco metálico no cilindro e realizado o traçado na radiografia, detectou-se em 13 situaçóes (65\%) o mesmo índice, isto é, 750 de curvatura o que, aliás, confere a este gabarito um bom indicador na determinação do ângulo de curvatura do canal simulado.

Como o objetivo deste trabalho é também determinar, por meio de traçado, 750 de curvatura após a obtenção do arco metálico, entende-se que esse percentual de acerto constitui método válido, porquanto os ângulos numericamente diferentes obtidos em 15\% dos casos foram de ângulos com $74^{\circ}$ de curvatura, valor muito próximo ao índice objetivado neste estudo, representando um grau (10) de diferença fato este também evidenciado na amostra número 16 com 760 de curvatura. Outro resultado apontado com diferença, pequena entre os ângu- los pretendidos após a obtenção do arco metálico foi das amostras 8, 11 e 19, tendo as duas primeiras a diferença de $5^{0}$ e a última dois graus $\left(2^{\circ}\right)$.

Por outro lado, pequenas diferenças numéricas, ou seja, $45 \%$ das ocorrências, apontam o mesmo grau de curvatura tanto antes como depois da inclusão dos arcos na unidade de embutimento em 9 casos, em $45 \%$ das medidas tomadas dos ângulos antes e depois, um grau (10) de diferença em 9 situaçóes, e em dois casos (10\%), a diferença foi de dois graus $\left(2^{\circ}\right)$ e cinco graus $\left(5^{\circ}\right)$.

$\mathrm{Na}$ verdade poucas foram às situaçóes de valores muito distantes em relação ao valor pretendido, ou seja, $75^{\circ}$, que foram os traçados realizados nas amostras 8 e 11 com ângulos $80^{\circ}(5,0 \%)$ e $70^{\circ}(5,0 \%)$, respectivamente.

Ora, o que se esperava era um cilindro que reproduzisse amostras com todos os ângulos iguais, já que foi construído a partir de cálculo trigonométrico, e se esperava um dispositivo fidedigno por se tratar de medida que levou à elaboração das equaçóes aqui demonstradas na metodologia chegando-se a esse cilindro com dimensões matemáticas precisas.

Apesar desses resultados, acredita-se que uma provável particularidade possa ter influenciado a determinação da curvatura do fio ortodôntico de aço inoxidável no ato da confecção do cilindro, a saber: flexibilidade do fio no momento em que foi moldado no cilindro, passível de não permanecer com mesmo ângulo que foi determinado; memória elástica de forma do fio supóe tendência deste material em voltar a sua posição original em maior ou menor grau, fato este evidenciado com maior tendência nas amostras com ângulos de $80^{\circ}$ e $70^{\circ}$, enquanto menor tendência foi ressaltada nas amostras com ângulos de $76^{\circ}, 74^{\circ} \mathrm{e} 73^{\circ}$.

Há diversas justificativas aceitáveis para justificar tais diferenças, ou seja, reação físico-química que envolve todo este processo e alta temperatura envolvida na tomada de presa da resina fenólica, expansão ou contração do fio metálico, inserção do pó de resina sobre o fio ortodôntico alterando o seu posicionamento, imprecisão na obtenção do ângulo do fio ortodôntico e imprecisão do traçado do arco.

Em relação à reação físico-química que envolve todo o processo, sabe-se que, durante a tomada de presa, os blocos envolvem ao mesmo tempo aquecimento e resfriamento do pó da resina com o objetivo de produzir a solidificação da substância. Graças à ocorrência desta reação, é possível que ocorram alteraçóes no fio ortodôn- 
Medeiros JMF, Rodrigues GA, Santos ACM, Rosa LCL, Carvalho PL, Nohara EL. Determinação do comprimento e grau de curvatura em canais radiculares curvos simulados confeccionados em blocos de resina fenólica. Revista de Odontologia da Universidade Cidade de São Paulo 2009 set-dez; 21(3): 202-11

tico, embora boa parte das amostras permanece como estava, ou seja, o mesmo grau de curvatura foi obtido.

Náo se ignora o fato de que o alto aquecimento produzido nessa fase de solidificação do bloco possa determinar no arco metálico contração ou expansão do fio ortodôntico, daí a razão de aumento ou diminuição do grau de curvatura dessas amostras.

Ademais, a inserção do fio ortodôntico sobre a primeira medida do pó de resina pode ter sido alterada na sua dimensão depois da segunda inserção do pó de resina sobre o fio ortodôntico arqueado provocando mudança de seu posicionamento original no interior da unidade de embutimento. Assim, os arcos inseridos nos blocos depois de preparados poderão apresentar uma posição diferente e que náo reflete o posicionamento exato depois da tomada radiográfica.

Mais ainda, tais diferenças podem ter ocorrido durante a obtenção do traçado do ângulo, tarefa esta realizada com auxílio de lápis, régua e transferidor com vistas à obtenção do grau de curvatura. Talvez essa dificuldade de traçado no papel vegetal tenha uma justificativa, uma vez que, durante a adaptação do fio ortodôntico no cilindro, quando da obtenção do arco, confeccionou-se uma curvatura cujo início em uma extremidade já é por demais curva e que termina na outra extremidade com a mesma característica, ou seja, também por demais curva, diferente, é claro, do modelo desenhado por Schneider ${ }^{31}$ (1971). Por essa razão, ao realizar-se o traçado antes e depois da inclusão, utilizou-se apenas uma pequeníssima extensão da parte reta do arco curvo, porquanto o eixo reto do canal curvo em sua extremidade onde se realizou o traçado é demasiadamente pequeno, daí tal dificuldade encontrada.

Alguns resultados foram imprevistos em alguns ca- sos, pois tais diferenças dos ângulos obtidos podem ter sido causadas durante a moldagem do fio no cilindro e, por outro lado, durante o traçado do arco na radiografia. Apesar disso, supóe-se que tal dispositivo representado por esse cilindro foi capaz de determinar o grau de curvatura desses arcos incluídos ou não. Por essa razão, tal modelo é uma ferramenta precisa e reproduzível.

Não se trata de recurso absoluto de determinaçáo do grau de curvatura, mas certamente refletiu muito bem, uma vez que o dispositivo que foi construído baseado em cálculo matemático reproduzindo no fio ortodôntico um arco cujo grau de curvatura ficou próximo ao desejado.

\section{CONCLUSÕES}

Diante do anteriormente exposto e dentro das condiçōes do presente experimento, parece válido concluir que a obtenção do arco metálico no cilindro nas duas condiçóes bem como o traçado para determinação do ângulo na radiografia representam uma ferramenta reproduzível e a avaliaçáo do processo de determinaçáo do grau de curvatura das amostras aponta que o método é apropriado, podendo ser avaliado e representar procedimento válido na determinação do grau de curvatura.

\section{AGRADECIMENTOS}

Agradecemos ao Professor Benedito Sergio Tavares Alvarenga pela segura orientação dos cálculos matemáticos e ao Técnico do Laboratório de Materiais e Ensaios e Nanotecnologia do Departamento de Engenharia Mecânica da Universidade de Taubaté, Sr. José Arauto Ribeiro, por todo o apoio e empenho demonstrado durante a etapa de confecção dos modelos. 
Medeiros JMF, Rodrigues GA, Santos ACM, Rosa LCL, Carvalho PL, Nohara EL. Determinação do comprimento e grau de curvatura em canais radiculares curvos simulados confeccionados em blocos de resina fenólica. Revista de Odontologia da Universidade Cidade de São Paulo 2009 set-dez; 21(3): 202-11

\section{REFERÊNCIAS}

1. Alkmin ST, Pinto CA, Habitante SM, Zöllner NA, Medeiros JMF, Lage-Marques JL. Concepção de um modelo experimental à base de resina fenólica utilizado em endodontia. In: $24^{a}$ Reunião Anual da Sociedade Brasileira de Pesquisa Odontológica; 2007; set; Atibaia. São Paulo: SBPqO; 2007.

2. Al-Sudani $\mathrm{D}, \mathrm{Al}-$ Shahrani S. A comparison of the canal centering ability of ProFile, K3, and RaCe Nickel Titanium rotary systems. J Endod, 2006 Dec; 32(12):1198-201.

3. Alves-Claro AP, Claro FA, Uzumaki ET. Wear resistance of nickel-titanium endodontic files after surface treatment. J Materials Scie: Materials Med, 2008 Oct; 19(10):3273-7.

4. Ankrum MT, Hartwell GR, Truitt JE. K3 Endo, Pro- Taper, and ProFile systems: breakage and distortion in severely curved roots of molars. J Endod, 2004 Apr; 30(4):234-7.

5. Anusavice KJ, Antonson SA. Materiais de acabamento e polimento. In: Anusavice KJ. Phillips Materiais dentários. 11 ed. Rio de Janeiro: Elsevier; 2005. p.337-39.

6. Borges, TF. Avaliação da capacidade de corte de limas de seção reta transversal quadrangular e triangular em liga de aço inoxidável. [Mestrado] Taubaté: Departamento de Odontologia da Universidade de Taubaté; 2005.

7. Calberson FLG, Deroose CAJG, Hommez GMG, De Moor RJG. Shaping ability of ProTaper nickeltitanium files in simulated resin root canals. Int Endod J, 2004 Set; 37(9):613-23.

8. Claro FAE. Avaliação do desempenho de limas endodônticas de níquel-titânio após tratamento de superfície. [Mestrado] Taubaté: Departamento de Engenharia Mecânica da Universidade de Taubaté; 2004.

9. Fariniuk, LF, Baratto-Filho, F, Guerisoli, DM, Barbizan, JV, Pedora, JD, Souza-Neto, MD. Modeling capacity of ENDOflash files in simulated root canals. Braz Dent J, 2001; 12(1):39-42.

10. Glosson, CR, Haller, RH, Dove, B, Del Rio, CE. A comparison of root canal preparations using $\mathrm{Ni}$-Ti hand, Ni-Ti engine-driven, and K-flex endodontic instruments. J Endod, 1995 Mar; 21(3):146-51.
11. Gonçalves, EMB. Análise da capacidade de corte e deformação das limas K\# e RT Densell. [Mestrado] Taubaté: Departamento de Odontologia da Universidade de Taubaté; 2007.

12. Grande NM, Plotino G, Pecci R, Bedini R, Malagnino VA, Somma F. Cyclic fatigue resistance and three-dimensional analysis of instruments from two nickel-titanium rotary systems. Int Endod J, 2006 Oct; 39(10): 755-763.

13. Guelzow A, Stamm O, Martus P, Kielbassa AM. Comparative study of six rotary nickel-titanium systems and hand instrumentation for root canal preparation. Int Endod J, 2005 Oct; 38(10):743-752.

14. Hulsmann M, Gressmann G, Schäfers F. A comparative study of root canal preparation using FlexMaster and HERO 642 rotary Ni-Ti instruments. Int Endod J, 2003 May; 36(5):358-366.

15. Hulsmann M, Herbst U, Schäfers F. Comparative study of root-canal preparation using Lightspeed and Quantec SC rotary NiTi instruments. Int Endod J, 2003 Nov; 36(11):748-756.

16. Javaheri HH, Javaheri GH. A comparison of three NI-TI rotary instruments in apical transportation. $J$ Endod, 2007 Mar; 33(3):284-286.

17. Johnson E, Lloyd A, Kuttler S, Namerow K. Comparison between a novel nickel-titanium alloy and 508 nitinol on the cyclic fatigue life of ProFile 25/.04 rotary instruments. J Endod, 2008 Nov; 34(11):1406-9. Epub 2008 sep 19.

18. Mahran AH, AboEl-Fotouh MM. Comparison of effects of ProTaper, HeroShaper, and Gates Glidden burs on cervical dentin thickness and root canal volume by using multislice computed tomography. J Endod, 2008 Oct; 34(10): 1219-1222. Epub 2008 Aug 23.

19. Manual de operação e manutenção. Pantec. Prensa hidráulica para embutimento de amostras metalográficas. Mod. Tempopress 2. São Paulo: Panambra Industrial e Técnica SA; 1992.

20. Medeiros JMF, Ishimoto NA, Alkmin ST, Carvalho PL, Risso VA, Zöllner NA. Eficiência de corte da lima de secção triangular usada manualmente e acoplada ao sistema rotatório Endo-Gripper. Publicatio UEPG Ci. Biol. Saúde, 2006 dez; 12(4):41-50. 
Medeiros JMF, Rodrigues GA, Santos ACM, Rosa LCL, Carvalho PL, Nohara EL. Determinação do comprimento e grau de curvatura em canais radiculares curvos simulados confeccionados em blocos de resina fenólica. Revista de Odontologia da Universidade Cidade de São Paulo 2009 set-dez; 21(3): 202-11

21. Medeiros JMF, Lima JP, Bombana AC. Analysis by scanning electron microscopy of the cutting surface changes on endodontics files according two methods for sterilizing and number of use. Act Report/ Brazilian Synchrotron Light Laboratory 2002; 6:267268.

22. Medeiros JMF, Risso VA, Haddad Filho MS, Carvalho PL, Zöllner NA. Morfologia da preparação de canais radiculares curvos com limas Flex- $\mathrm{R}$ manual e acoplada ao sistema Endo-Gripper. Saúde Oral Rev Port de Estomatol e Méd Dent, 2006 set./out; 50(5):5262.

23. Medeiros JMF, Simi Jr J, Risso VA. Estudo comparativo mediante pesagem de duas técnicas de preparo de canais radiculares curvos com um tipo de instrumento endodôntico. Rev. Odontol. UNAERP, 2000 jan./dez; 3(1):11-16.

24. Medeiros JMF, Zöllner NA, Carvalho PL, Alves APR, Clemente RGP. Capacidad de corte de la lima Flexofile en canales simulados. Rev Cubana Estomatol, 2008 Ene/Mar; 45(1): 1-2.

25. Morrinson SW, Newton CW, Brown Jr CE. The effects of steam sterilization and usage on cutting efficiency of endodontic instruments. J Endod, 1989 Sep; 15(9):427-31.

26. Plotino G, Grande NM, Sorci E, Malagnino VA, Somma F. A comparison of cyclic fatigue between used and new Mtwo Ni-Ti rotary instruments. Int Endod J, 2006 Sep; 39(9):716-23.

27. Plotino G, Grande NM, Sorci E, Malagnino VA, Somma F. Influence of a brushing working motion on the fatigue life of NiTi rotary instruments. Int Endod J, 2007 Jan; 40(1):45-51.

28. Sakane, FK. Avaliação in vitro do desgaste e da capacidade de corte de limas manuais de NI-TI. [Mestrado] Taubaté: Departamento de Odontologia da Universidade de Taubaté; 2007.

29. Schäfer E, Tepel J. Cutting efficiency of Hedstrom, $\mathrm{S}$ and $\mathrm{U}$ files made of various alloys in filing motion. Int Endod J, 1996 Sep; 29(5):302-8.

30. Schäfer, E. Effects of four instrumentation techniques on curved canals: a comparison study. $J$ Endod, 1996 Dec; 22(12): 685-9.
31. Schneider, S.W. A comparison of canal preparations in straight and curved root canals. Oral Surg Oral Med Oral Patol, 1971 Aug; 2(32):271-5.

32. Silva EAB. Avaliação comparativa da capacidade e perda de corte de limas endodônticas tipo $\mathrm{K}$ de aço inoxidável (Flexofile) e de níquel-titânio (Nitiflex). [Mestrado] Taubaté: Departamento de Odontologia da Universidade de Taubaté; 2001.

33. Song YL, Bian Z, Fan B, Fan MW, Gutmann JL, Peng B. A comparison of instrument-centering ability within the root canal for three contemporary instrumentation techniques. Int Endod J, 2004 Apr; 37(4):265-271.

34. Souza V, Barbosa HG, Holland R, Dezan Junior E, Nery MJ, Ottoboni Filho JA, et al. Eficiência de corte alguns tipos de limas endodônticas. Rev Ciências Odontol, 1998; 1(1):65-74.

35. Szep S, Gerhardt T, Leitzbach C, Luder W, Heidemann D. Preparation of severely curved simulated root canals using engine-driven rotary and conventional hand instruments. Clin Oral Investig, 2001 Mar; 5(1):17-25.

36. Veltri M, Mollo A, Mantovani L, Pini P, Balleri S, Grandini S. A comparative study of Endoflare-Hero Shaper and Mtwo NiTi instruments in the preparation of curved root canals. Int Endod J, 2005 Sep; 38(9):610- 6.

37. Veltri, M. Mollo A, Pini PP, Ghelli LF, Balleri P. In vitro: comparison o shaping abilities of ProTaper and GT rotary files. J Endod, 2004 Mar; 30(3):163-6.

38. Yang GB, Zhou XD, Zheng YL, Zhang H, Shu Y, Wu HK. Shaping ability of progressive versus constant taper instruments in curved root canals of extracted teeth. Int Endod J, 2007 Sep; 40(9):707-14. Epub 2007 Jul 23.

39. Yoshimine $\mathrm{Y}$, Ono M, Akamine A. The shaping effects of three nickel-titanium rotary instruments in simulated S-shaped canals. J Endod, 2005 May; 31(5):373-5.

Recebido em: 23/3/2009

Aceito em: 1/10/2009 